Article

\title{
Spectroscopic and Photometric Variability of Three Oxygen Rich Post-AGB "Shell" Objects
}

\author{
Griet C. Van de Steene ${ }^{1, *(\mathbb{D})}$, Bruce J. Hrivnak ${ }^{2}\left(\mathbb{D}\right.$, Hans $_{\text {Van }}$ Winckel $^{3}\left(\mathbb{D}\right.$, Julius Sperauskas ${ }^{4}(\mathbb{D})$ \\ and David Bohlender 5 (iD \\ 1 Royal Observatory of Belgium, Astronomy and Astrophysics, Ringlaan 3, 1180 Brussels, Belgium \\ 2 Department of Physics and Astronomy, Valparaiso University, Valparaiso, IN 46383, USA; \\ Bruce.Hrivnak@valpo.edu \\ 3 Instituut voor Sterrenkunde, K.U. Leuven University, Celestijnenlaan 200 D, B-3001 Leuven, Belgium; \\ h.vanwinckel@kuleuven.ac.be \\ 4 Institute of Theoretical Physics and Astronomy, Vilnius University Observatory, Ciurlionio 29, 2009 Vilnius, \\ Lithuania; julius.sperauskas@ff.vu.lt \\ 5 National Research Council of Canada, Herzberg Astronomy and Astrophysics, 5071 West Saanich Road, \\ Victoria, BC V9E 2E7, Canada; david.bohlender@nrc-cnrc.gc.ca \\ * Correspondence: g.vandesteene@oma.be
}

Received: 28 June 2018; Accepted: 29 November 2018; Published: 3 December 2018

\begin{abstract}
Light, color, and radial velocity data (2007-2015) for HD 161796, V887 Her, and HD 331319, three oxygen-rich post-AGB stars, have thus far not provided direct support for the binary hypothesis to explain the shapes of planetary nebulae and severely constrain the properties of any such undetected companions. The light and velocity curves are complex, showing similar periods and variable amplitudes. Nevertheless, over limited time intervals, we compared the phasing of each. The color curves appear to peak with or slightly after the light curves, while the radial velocity curves peak about a quarter of a cycle before the light curves. Thus it appears that these post-AGB stars are brightest when smallest and hottest. The spectra of these objects are highly variable. The $\mathrm{H} \alpha$ line has multiple, variable emission and absorption components. In these oxygen-rich post-AGB stars atmospheric lines, such as near-infrared Ca II triplet and low-excitation atomic lines, also have multiple components and sometimes show line doubling, indicative of shocks induced by pulsation.
\end{abstract}

Keywords: late-stage stellar evolution; planetary nebulae; multi-wavelength photometry; radial velocity; stellar evolution; pulsation; shock wave

\section{Introduction}

Stars at the proto-planetary nebula (PPN) phase evolve from the Asymptotic Giant Branch towards the PN phase becoming hotter at almost constant luminosity. These low mass stars are surrounded by a circumstellar envelope ejected during the AGB phase. The details of the physical processes and stellar winds that shape these envelopes remain uncertain. The outstanding question in PN research is the role and importance of binarity in the shaping of PNe.

We selected seven bright $(\mathrm{V}<10 \mathrm{mag}$ ) post-AGB stars observable with 1-m class telescopes from the northern hemisphere (Hrivnak et al. [1]). They all have F- and G spectral types. Of these 7 metal-poor PPNe, 3 are O-rich and 4 are C-rich. In this contribution, we will consider the 3 O-rich objects: HD 161796 (IRAS 17436+5003), HD 331319 (IRAS 19475+3119), and V887 Her (IRAS 18095+2704). The objects have spectral energy distributions typical for "shell" objects: they are double peaked, with a peak in the visible arising from the (reddened) photosphere and a second peak in the mid-infrared arising from re-radiation from $\operatorname{cool}(\mathrm{T} \leq 200 \mathrm{~K})$ dust. However, they have no 
near-infrared excess, the presence of which is an indication of the presence of a disk. The disk-type post-AGB stars have been shown to be binaries (Manick et al. [2], Van Winckel et al. [3]).

HD 161796 is highly aspherical, and shows a low density polar outflow and a high density equatorial region (Min et al. [4]). HD 331319 is a quadrupolar nebula with two collimated bipolar lobes (Sahai et al. [5]). V887 Her is an extended source with a significant circumstellar shell (Ueta et al. [6]) and $\mathrm{OH}$ masers (Lewis et al. [7]).

To prove or exclude the presence of binaries in these shell objects is crucial to our understanding of the way(s) that bipolar PPNe and PNe form. If it turns out that these PPNe are not binaries, then it raises the possibility that there may be more than one way to produce bipolar PNe.

\section{Observations and Analysis}

High-resolution spectra were obtained at the Dominion Astrophysical Observatory $1.2 \mathrm{~m}$ telescope since 2007 and with the HERMES Spectrograph on the $1.2 \mathrm{~m}$ Mercator telescope (Raskin et al. [8], Van Winckel et al. [9]) since 2009. Radial velocities of the HERMES spectra were determined via the cross-correlation technique using a mask in the range $477-655 \mathrm{~nm}$ for F0-type stars and fitting the peak of the obtained correlation function with a gaussian (Hrivnak et al. [1]). Radial velocities of DAO spectra were also obtained via cross correlation with a set of bright IAU RV standards observed with the same instrumentation. In this case velocities were determined by fitting a parabola to the upper half of the cross correlation profiles (Hrivnak et al. [1]).

Photometric observations of these objects were obtained from 2007 to 2015 at the Valparaiso University Observatory with a $0.4 \mathrm{~m}$ telescope equipped with a CCD camera and filters standardized to the Johnson B and V and Cousins R systems. Differential photometry was employed, using an aperture of $11^{\prime \prime}$ diameter (Hrivnak et al. [10]). For V887 Her ASAS-N data were available and used (Kochanek et al. [11]).

\section{Results and Discussion}

\subsection{Radial Velocity and Photometry}

The RV amplitudes are small $(<4.0 \mathrm{~km} / \mathrm{s}$, Hrivnak et al. [12]) and the periods found in the RV data are in the range 35 to 103 days, similar to the pulsation periods of the objects. So far, no clear evidence for long-period RV variations due to binary companions has been found in any of these objects. This sets significant constraints on the properties of any undetected binary companion: they must be of low mass, $\leq 0.2 \mathrm{M}_{\odot}$, or long period, longer than 30 years. Thus the present observations do not provide direct support for the binary hypothesis to explain the shapes of thes PPNe.

The light and velocity curves are complex with multiple periods and variable amplitudes. However, over limited time intervals during 2007-2015, we were able to identify dominant periods in the light, color, and velocity curves and compare the phasing of each. See figures in Hrivnak et al. [12] which shows the phased light, color, and velocity curves of the objects. The color curves appear to peak with or slightly after the light curves while the RV curves peak about a quarter of a cycle before the light curves. For all three objects plus two previously studies PPNe (Hrivnak et al. [13]), (a) the light and color curves are approximately in phase, with the suggestion that the color curve perhaps peaks slightly $(\sim 0.05 \mathrm{P})$ after the light. (b) The RV curve is approximately $-0.25 \mathrm{P}$ out of phase with the light curve. Thus it appears that these PPNe are brightest when smallest and hottest. These results differ from those found for Cepheid variables, where the light and velocity differ by half a cycle, and are hottest at about average size and expanding. However, they do appear to have similar phasing to the larger amplitude pulsations seen in RV Tauri variables. Attempts at modeling pulsation in these post-AGB stars are few (Fokin et al. [14]) and the observed periods are longer than models indicate. 


\subsection{Spectroscopic Variability}

The average $\mathrm{H} \alpha$ profiles show broad wings with central emission and absorption components (Figure 1). The individual spectra show that these $\mathrm{H} \alpha$ components vary in depth, in width, and position (Figure 2). The strength of the blue and red emission are changing alternately. The red emission and absorption component is generally weaker than the blue. In the inverse P-Cygni profile phase, the blue emission peak can be very strong.
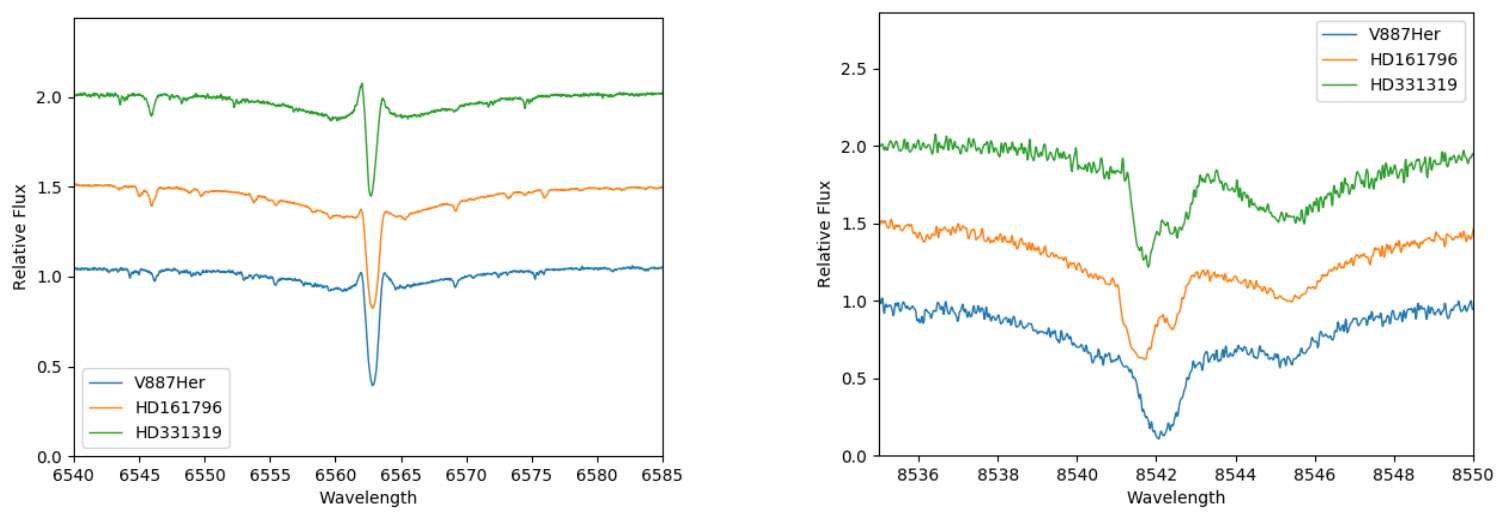

Figure 1. (left) The averaged $\mathrm{H} \alpha$ profiles of all spectra corrected for RV and normalized. (right) The averaged Ca II $854.2 \mathrm{~nm}$ profiles of all spectra corrected for RV and normalized.

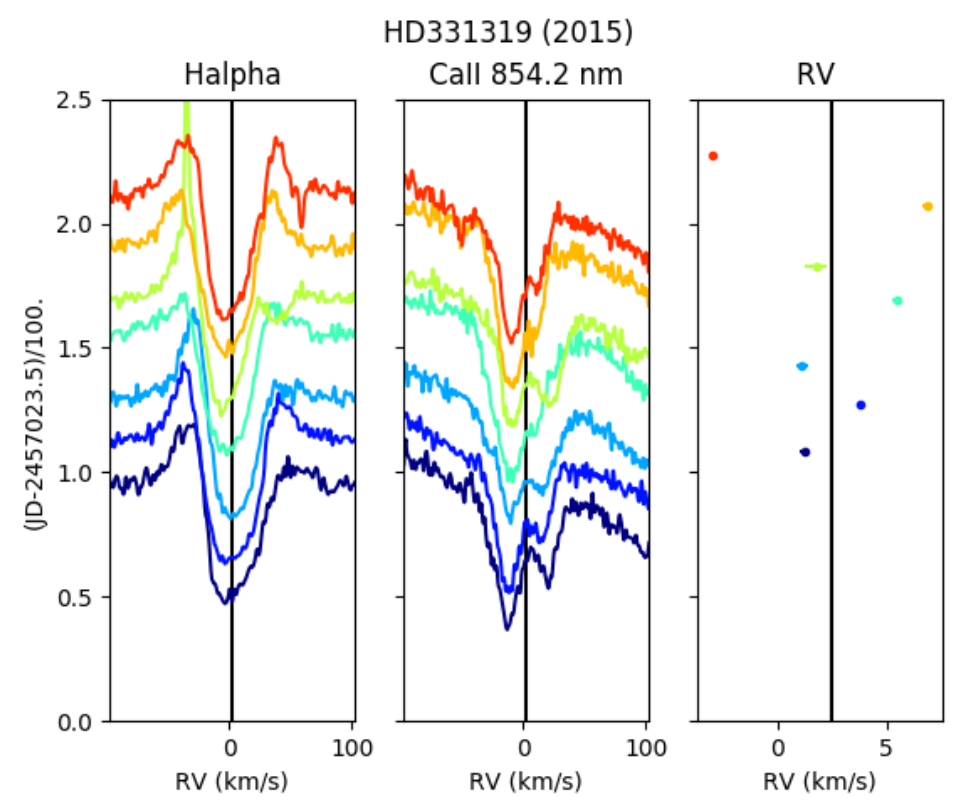

Figure 2. HD 331319 line profiles observed in 2015. At the left $\mathrm{H} \alpha$, in the middle the Ca II line at $\lambda 854.2 \mathrm{~nm}$, and at the right the RV's measured in the spectrum. The y-axes are Julian Dates, modified as indicated.

The near-infrared Ca II triplet and some low-excitation metal lines such as Ba II and Fe II also show strong variability (Figure 2). In these lines, line splitting in two can be observed sometimes. This splitting was observed in C-rich post-AGB stars (Klochkova [15]) with a complex circumstellar envelope, but here we confirm that it is also observed in such O-rich post-AGB stars. Further analysis will help clarify where these components originate in these stars. The observed splitting and short-term variability in low excitation atomic lines provide evidence that shock waves are present in the outer layers of the atmosphere or circumstellar envelope (Fokin et al. [14], Klochkova [15], Zacs et al. [16]). 
The lack of line splitting phenomena in the high excitation potential lines is most likely because these lines are formed deeper in the atmosphere.

The complex spectral variability and lack of stable periods in the light and velocity curves makes the temporal changes hard to phase. Provisionally, in Figure 3 we phased V887Her to the period of 102.3 days and the same epoch as Hrivnak et al. [12]. We searched for evidence for a jet as seen in BD+46442 (Bollen et al. [17]), in which a broad absorption with a velocity up to $\sim-400 \mathrm{~km} / \mathrm{s}$ is observed. In the phased spectra of V887 Her there is an extended blue absorption at phase 0.35 . However the velocity at the blue edge of the absorption is only $-50 \mathrm{~km} / \mathrm{s}$, no more than 2-3 times the typical expansion velocity of PNe and AGB stars, and lasts less than 0.10 phase cycle, while in BD+46 442 it is at least 0.25 phase cycle. These are not indications for a strong jet. These "Shell" objects have $\mathrm{H} \alpha$ profiles similar to "disc" sources, but without the presence of strong jets.

The analysis of the high-resolution spectra of these post-AGB stars continues, in order to better investigate and understand the shocks, pulsation, and kinematics in these objects.

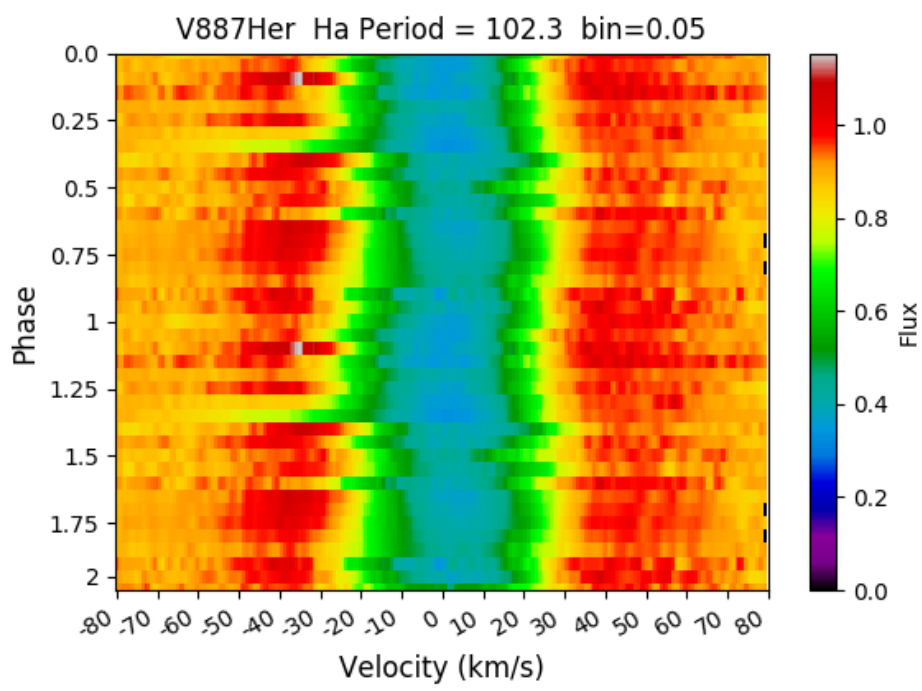

Figure 3. Dynamic H $\alpha$ spectra of V887 Her, phased to 102.3 days and binned to 0.05 , showing different phase-dependent variations in the line profiles. The velocity on the $\mathrm{x}$-axis denotes the RV shift from the laboratory wavelength. The color indicates the continuum normalized flux.

Author Contributions: G.C.V.d.S.: obtained the data, did data analysis and data reduction, and wrote the paper B.J.H. obtained data, did data reduction and analysis, and helped writing up the paper. H.V.W., J.S., and D.B. contributed to the spectroscopic and/or photometric observations.

Funding: B.J.H. acknowledges ongoing support from the National Science Foundation (most recently AST 1009974, 1413660). J.S. acknowledge support of the Research Council of Lithuania under the grant MIP-085/2012. This research has been conducted in part based on funding from the Research Council of K.U. Leuven (GOA/13/012) and was partially funded by the Belgian Science Policy Office under contract BR/143/A2/STARLAB.

Acknowledgments: This research has made use of the SIMBAD database, operated at CDS, Strasbourg, France, and NASA's Astrophysical Data System. We also thank the many Valparaiso University undergraduate students and colleagues at the ROB, KUL, and ULB who obtained observations for the project.

Conflicts of Interest: The authors declare no conflict of interest.

\section{References}

1. Hrivnak, B.J.; Van de Steene, G.; Van Winckel, H.; Sperauskas, J.; Bohlender, D.; Lu, W. Where are the Binaries? Results of a Long-term Search for Radial Velocity Binaries in Proto-planetary Nebulae. Astrophys. J. 2017, 846, 96. [CrossRef]

2. Manick, R.; Van Winckel, H.; Kamath, D.; Hillen, M.; Escorza, A. Establishing binarity amongst Galactic RV Tauri stars with a disc. Astron. Astrophys. 2017, 597, A129. [CrossRef] 
3. Van Winckel, H. Post-AGB binaries as tracers of stellar evolution. In Planetary Nebulae: Multi-Wavelength Probes of Stellar and Galactic Evolution; Liu, X., Stanghellini, L., Karakas, A., Eds.; IAU Symposium; International Astronomical Union: Paris, France, 2017; Volume 323, pp. 231-234. [CrossRef]

4. Min, M.; Jeffers, S.V.; Canovas, H.; Rodenhuis, M.; Keller, C.U.; Waters, L.B.F.M. The color dependent morphology of the post-AGB star HD 161796. Astron. Astrophys. 2013, 554, A15. [CrossRef]

5. Sahai, R.; Sánchez Contreras, C.; Morris, M.; Claussen, M. A Quadrupolar Preplanetary Nebula: IRAS 19475+3119. Astrophys. J. 2007, 658, 410-422. [CrossRef]

6. Ueta, T.; Meixner, M.; Bobrowsky, M. A Hubble Space Telescope Snapshot Survey of Proto-Planetary Nebula Candidates: Two Types of Axisymmetric Reflection Nebulosities. Astrophys. J. 2000, 528, 861-884. [CrossRef]

7. Lewis, B.M. On the Transience of Circumstellar Shells about $|\mathrm{B}|>=10 \mathrm{deg}$ OH/IR Stars. I. Basic Statistics. Astrophys. J. 2000, 533, 959-968. [CrossRef]

8. Raskin, G.; van Winckel, H.; Hensberge, H.; Jorissen, A.; Lehmann, H.; Waelkens, C.; Avila, G.; de Cuyper, J.P.; Degroote, P.; Dubosson, R.; et al. HERMES: A high-resolution fibre-fed spectrograph for the Mercator telescope. Astron. Astrophys. 2011, 526, A69. [CrossRef]

9. Van Winckel, H.; Jorissen, A.; Gorlova, N.; Dermine, T.; Exter, K.; Masseron, T.; Østensen, R.; Van Eck, S.; Van de Steene, G. Post-AGB binaries in an evolutionary perspective: A HERMES monitoring programme. Memorie della Societa Astronomica Italiana 2010, 81, 1022.

10. Hrivnak, B.J.; Lu, W.; Nault, K.A. Variability in Proto-planetary Nebulae. IV. Light Curve Analysis of Four Oxygen-rich, F Spectral Type Objects. Astron. J. 2015, 149, 184. [CrossRef]

11. Kochanek, C.S.; Shappee, B.J.; Stanek, K.Z.; Holoien, T.W.S.; Thompson, T.A.; Prieto, J.L.; Dong, S.; Shields, J.V.; Will, D.; Britt, C.; et al. The All-Sky Automated Survey for Supernovae (ASAS-SN) Light Curve Server v1.0. Publ. Astron. Soc. Pac. 2017, 129, 104502. [CrossRef]

12. Hrivnak, B.J.; Van de Steene, G.; Van Winckel, H.; Lu, W.; Sperauskas, J. Variability in Proto-Planetary Nebulae: V. Velocity and Light Curve Analyses of IRAS 17436+5003, 18095+2704, and 19475+3119. arXiv 2018, arXiv:astro-ph.SR/1810.13037.

13. Hrivnak, B.J.; Lu, W.; Sperauskas, J.; Van Winckel, H.; Bohlender, D.; Začs, L. Studies of Variability in Proto-planetary Nebulae. II. Light and Velocity Curve Analyses of IRAS 22272+5435 and 22223+4327. Astrophys. J. 2013, 766, 116. [CrossRef]

14. Fokin, A.B.; Lèbre, A.; Le Coroller, H.; Gillet, D. Non-linear radiative models of post-AGB stars: Application to HD 56126. Astron. Astrophys. 2001, 378, 546-555. [CrossRef]

15. Klochkova, V.G. Circumstellar envelope manifestations in the optical spectra of evolved stars. Astrophys. Bull. 2014, 69, 279-295. [CrossRef]

16. Začs, L.; Musaev, F.; Kaminsky, B.; Pavlenko, Y.; Grankina, A.; Sperauskas, J.; Hrivnak, B.J. Spectroscopic Variability of IRAS 22272+5435. Astrophys. J. 2016, 816, 3. [CrossRef]

17. Bollen, D.; Van Winckel, H.; Kamath, D. Jet creation in post-AGB binaries: the circum-companion accretion disk around BD+46 442. Astron. Astrophys. 2017, 607, A60. [CrossRef]

(C) 2018 by the authors. Licensee MDPI, Basel, Switzerland. This article is an open access article distributed under the terms and conditions of the Creative Commons Attribution (CC BY) license (http://creativecommons.org/licenses/by/4.0/). 